Documents pour l'histoire du français langue étrangère ou seconde

$44 \mid 2010$

De l'École de préparation des professeurs de français à l'étranger à l'UFR DFLE. Histoire d'une institution (1920-2008)

\title{
Le couple Fouché-Mercier ou l'art de la maïeutique
}

Pierre Léon et Monique Léon

\section{(2) OpenEdition}

\section{Journals}

Édition électronique

URL : https://journals.openedition.org/dhfles/2753

DOI : $10.4000 /$ dhfles. 2753

ISSN : 2221-4038

\section{Éditeur}

Société Internationale pour l'Histoire du Français Langue Étrangère ou Seconde

Édition imprimée

Date de publication : 1 janvier 2010

Pagination : 79-87

ISSN : 0992-7654

Référence électronique

Pierre Léon et Monique Léon, « Le couple Fouché-Mercier ou l'art de la maïeutique », Documents pour I'histoire du français langue étrangère ou seconde [En ligne], 44 | 2010, mis en ligne le 31 janvier 2014, consulté le 28 mars 2023. URL : http://journals.openedition.org/dhfles/2753 ; DOI : https://doi.org/ $10.4000 /$ dhfles. 2753

Ce document a été généré automatiquement le 28 mars 2023.

Tous droits réservés 


\title{
Le couple Fouché-Mercier ou l'art de la maïeutique
}

\author{
Pierre Léon et Monique Léon
}

1 Nous les avons toujours associés, Fouché-Mercier. Lui était l'image même du directeur, autoritaire, sachant être affable, protecteur, et capable de passer du calme olympien à la colère de Zeus. Nous le craignions pour sa rigueur et l'admirions pour une science qu'il dispensait au compte-gouttes.

2 Elle était le portrait typique de l'assistante du Maître. Elle savait le flatter, l'amadouer, lui témoigner publiquement les éloges les plus flatteurs, nous rappelant dans son cours que Fouché était l'inspirateur et le guide à qui nous devions reconnaissance et respect.

\section{Image du Maître}

3 Pierre Fouché. Quand il arrivait dans l'amphithéâtre du cinquième étage de la Sorbonne, au 46 rue Saint-Jacques, le silence s'abattait sur nous. Il entrait, les mains derrière le dos, la tête légèrement penchée, montrant le crâne chauve du savant tel qu'on représentait le professeur Nimbus dans les caricatures de l'époque. Toujours élégant, complet, cravate, il montait à l'estrade de son pas souple et élastique de Pyrénéen. Nous devions savoir ainsi qu'il était resté jeune malgré sa calvitie.

4 Les autres professeurs arrivaient, les uns comme Lanson - le neveu de l'Autre - avec sa canne et son chapeau; les autres - comme Lamaison, avec un gros cahier, ou Charles Bruneau avec des notes qu'il extrayait une à une de toutes ses poches. Fouché avait la coquetterie de venir les mains vides. Il savait tout par cœur, du simple cours d'orthoépie élémentaire aux inextricables «Questions de linguistique romane» du jeudi soir, où quelques élus étaient admis, y compris trois thésards de taille : Pignon, Arveiller et Molho.

5 Il donnait aussi le « Cours de phonétique générale » du français, obligatoire pour ceux qui faisaient la licence, en même temps que le diplôme de l'École supérieure de préparation et de perfectionnement des professeurs de français à l'étranger (ESPPPFE) 
et, de plus, le cours destiné à ceux qui préparaient le diplôme d'études supérieures en phonétique avec Marguerite Durand, chargée de la phonétique expérimentale.

6 L'ESPPPFE avait certainement besoin, à ses débuts, d'une poigne solide et d'un bon jugement pour la diriger. Fouché avait été l'homme qu'il fallait. Son choix des enseignants avait été remarquable. La première année, il s'était octroyé le cours d'orthoépie, ce qui lui donnait visiblement le temps de faire ailleurs des recherches qu'il jugeait plus sérieuses. Il faut dire aussi que ce cours était exprès destiné aux étrangers et que les francophones qui devaient le suivre également étaient censés en tirer un profit pédagogique.

$7-$ «Écrivez ! » était la première injonction du maitre qui dictait SON cours d'orthoépie.

$8-$ «É accentué en Sssss... » (Là, il fallait dire : Syllabe), « Fffff... » (Il attendait : Fermée).

9 Il reprenait lentement en martelant chaque mot: «É... ACCENTUÉ... EN... SYLLABE... FERMÉE... EST... TOUJOURS... OU... OU... OU... » (Et là, il fallait crier tous ensemble : Ouvert!)

10 Et il répétait toute la règle, ajoutant l'exemple attendu, qui nous faisait sourire (intérieurement) : «FENÊTRE! » qu'il prononçait avec son tenace accent de Perpignan : [fənetrə] ou un bel E fermé remplaçait le E ouvert annoncé.

11 - «́́crivez!»

12 Les têtes se penchaient à nouveau sur les cahiers, tandis que lui circulait à travers les tables de l'amphithéâtre cherchant d'un œil inquisiteur le coupable qui aurait laissé un vide. Personne n'osait poser de question et comme le sujet était inconnu de tous, on était persuadé, les premières semaines, qu'il inventait tout pour nous à mesure qu'il parlait. En nous ouvrant les portes de son savoir phonétique, il attirait en même temps notre attention sur la réalité du français parlé, éveillant notre esprit critique peut-être plus encore quand ses exemples à couleur méridionale démentaient la règle énoncée. Il construisait, à pas mesurés, l'architecture linguistique de ce qu'on commençait à appeler «Structure» de la langue, avec ses oppositions, ses distributions complémentaires et tout ce que cela impliquait de vues nouvelles. Cependant, ignorant volontairement Saussure et ses disciples, refusant toute discussion théorique sur la phonologie que Martinet enseignait à côté, Fouché nous condamnait à sa seule science. Jamais une seule fois, dans son cours de première année, il n'a cité un autre phonéticien que lui, alors qu'il avait été l'élève de Grammont et qu'il connaissait bien les ouvrages de Passy.

Pour voir les choses positivement, disons que Fouché, avec sa rigueur méthodique et sa volonté de puissance, nous a d'abord donné l'envie d'en savoir plus!

14 Par contre, dans son cours pour le diplôme d'études supérieures, Fouché reprenait toutes les grandes lois phonétiques de Grammont et le citait parfois. Les noms d'auteurs allemands et espagnols tels que Meyer-Lübke et Menéndez Pidal, étaient évoqués de temps à autre, ajoutant une note de mystère savant. Lorsque fut créée la seconde année d'études à l'ESPPPFE, Fouché reprit le cours de première année en le développant quelque peu, y ajoutant en particulier une mince dimension prosodique. 


\section{De la philologie avant toute chose...}

15 Cependant, alors que les laboratoires de phonétique en Angleterre, en Allemagne, en Suède, au Danemark, en Allemagne et aux États-Unis se développaient, s'équipant de matériels nouveaux, pour des recherches acoustiques et physiologiques, Fouché ne s'en est vraiment jamais occupé. Il semblait allergique à la technologie et avait haussé les épaules quand Marguerite Durand était un jour descendue de son laboratoire enfumé pour venir nous parler des travaux de Delattre sur la synthèse de la parole. La pauvre devait bien souffrir avec son tambour de Rousselot qui inscrivait les vibrations de la parole transmise par une embouchure jusqu'au stylet, collé sur le caoutchouc d'un préservatif. Straka qui avait monté un laboratoire d'études ciné-radiographiques de la parole était dénigré par Fouché et lui rendait bien son mépris. Martinet, de son côté, ignorait les deux autres.

Le soir où Marguerite Durand était venue nous parler de la synthèse de la parole et des travaux de Delattre, elle avait amené avec elle Roman Jakobson, de passage à Paris, curieux de savoir ce qui se faisait comme recherches à l'Institut de phonétique et quelles applications pratiques on en faisait avec les étudiants étrangers de cette ESPPPFE, dont on commençait à parler. Jakobson était ami de Delattre et les deux hommes avaient une admiration réciproque. Fouché, qui était absorbé dans la philologie, ignorait royalement tous les aspects technologiques aussi bien que linguistiques des nouveautés américaines. Il fut courtois, sans plus.

Fouché avait, dans ses cours, le mérite de pratiquer l'art de la maïeutique. L'exemple cidessus, pour nous faire accoucher de la règle du $\mathrm{E}$ accentué en syllabe fermée, en est une illustration caricaturale. Il était épris de stylistique orale - que l'on n'appelait pas encore phonostylistique. Je lui dois d'avoir éveillé en moi la passion du rythme et de l'intonation. Il la devait lui-même à Maurice Grammont, dont il nous faisait retrouver les analyses phonostylistiques de textes littéraires, telle la célèbre période de Bossuet: "Celui qui règne dans les cieux, et de qui relèvent tous les empires, à qui seul appartient la gloire, la majesté et l'indépendance, est aussi le seul qui se permet de faire la loi aux rois et de leur donner, quand il lui plait, de grandes et de terribles leçons. » Nous devions en retrouver l'architecture rythmique et mélodique et en réciter ensemble le texte comme l'aurait fait un chœur dans une cathédrale.

Fouché avait aussi grande admiration pour Baudelaire, nous faisant comparer longuement le texte en prose de $L a$ Chevelure à celui du poème versifié. On le sentait inspiré, lorsqu'il déclamait religieusement "Sois sage, ô ma douleur et tiens-toi plus tranquille... » On a passé également bien des heures à disserter sur les trois derniers vers de ce poème, pour découvrir le jeu symbolique de leurs sonorités :

Le soleil moribond s'endormir sous une arche,

Et, comme un long linceul trainant à l'Orient,

Entends, ma chère, entends, la douce nuit qui marche.

19 Fouché faisait durer le plaisir, nous obligeant cent fois à retravailler une intonation qui aurait créé un faux-sens, ou un contresens, dans le texte. Puis, il nous le donnait à interpréter, chacun notre tour - en commentant parfois sèchement la réalisation. On a passé ainsi des semaines à dire et redire ce mince extrait de Loti : "Ils revenaient, les Islandais!» Mais Fouché, qui pourtant était bon musicien, restait intuitif pour l'analyse de l'aspect prosodique du langage, comme s'il avait été impossible de théoriser, en dehors de textes «bien formés », comme diront plus tard les linguistes générativistes. 
S'il avait éveillé notre attention à l'oral, il ignorait les problèmes de la langue parlée spontanée, de tous les jours, en dehors de ceux du E caduc et de la liaison. Il voyait d'un mauvais œil la naissance de la linguistique appliquée en France, et celle des laboratoires de langues.

Et pourtant, avec la direction de l'ESPPPFE, Fouché avait aussi celle de l'Institut de phonétique. Des deux côtés, il avait devant lui des étrangers venus surtout pour corriger leur accent et éventuellement apprendre les techniques de correction phonétique. Tout un personnel enseignant de dames, entraînées surtout à la diction poétique par des comédiens, donnait des cours dans cette vénérable institution. Je pense qu'elles ne s'en tiraient pas trop mal, comptant sur leur oreille. Et Fouché s'en satisfaisait.

\section{Portrait d'une remarquable praticienne}

Suzanne Mercier était, elle aussi, venue des cours de diction du théâtre et en portait la marque avec panache. Elle parlait souvent de son amie Dussane, sociétaire de la Comédie-Française et d'autres grands comédiens qu'elle aurait rencontrés au Conservatoire. Elle arrivait à notre cours de l'École, en courant, toujours un peu en retard. Tout en s'excusant, elle retenait, de la main gauche, une grande capeline - bleu pastel, comme le long manteau qu'elle ne quittait jamais. D'un geste large, elle posait sur le bureau un sac noir, mystérieux, que l'on imaginait contenir le matériel de maquillage pour son entrée en scène. Elle montait lentement sur l'estrade et commençait son spectacle par un silence, saluant son public d'un regard circulaire, accompagné d'un geste large, majestueux, et d'un sourire de diva. Et puis, elle descendait dans l'arène, enchaînant de la voix et du geste le poème de la fois précédente. Ou bien, lançant, comme une réplique attendue : « Mais oui, nous en étions au problème du $\mathrm{R}$ ! N'est-ce pas?»

22 Grande dame fort cultivée, elle ne manquait jamais de citer ses auteurs préférés, Mégret, le Père Bouhours, Gilles Vaudelin et avait toujours une bonne anecdote à propos d'une remarque de Vaugelas. Et puis commençait la remarquable séance de correction phonétique. Elle avait une excellente oreille, nous faisant découvrir, chez l'étranger, l'erreur de prononciation et la manière de la corriger. 


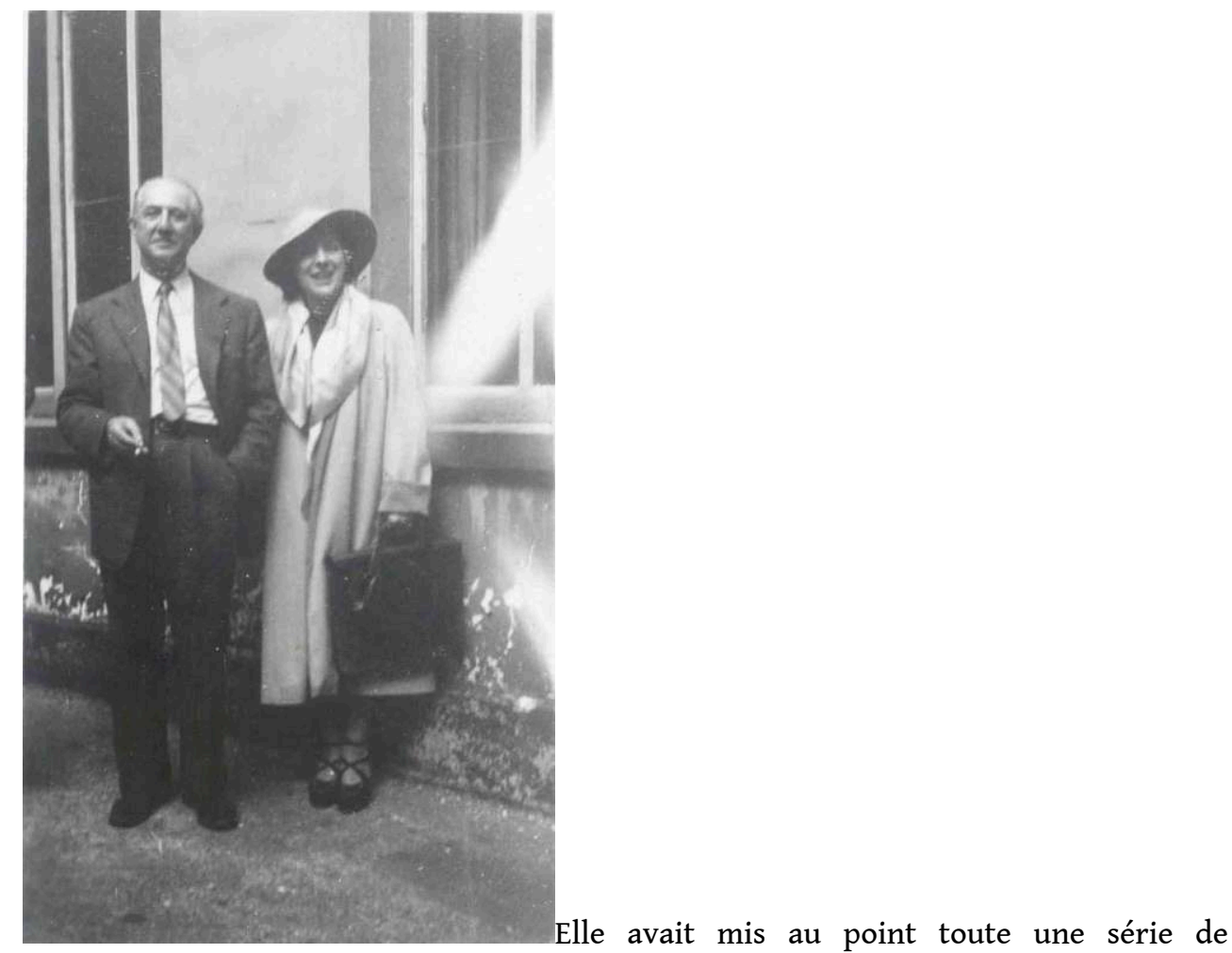
techniques articulatoires. L'une d'entre elles était l'utilisation de ce qu'elle appelait « les phonèmes professeurs ». Ainsi, ayant dessiné le diagramme de la prononciation du $\mathrm{R}$ dorso-uvulaire, elle faisait dire "ERKI » aux étudiants anglophones dont le R est prononcé avec la pointe de la langue relevée et le dos de la langue en creux. Le E et le I faisaient garder la langue contre le plancher du palais et le $\mathrm{K}$ obligeait la langue à garder, dans sa partie dorsale, la forme convexe, dorso-uvulaire du $\mathrm{R}$ français. Pour faciliter l'acquisition d'une voyelle nasale, elle faisait chanter l'orale correspondante sur une note donnée puis la faisait descendre brusquement d'un ton. Ça marchait !

Pierre Fouché et Suzanne Mercier.

Archives personnelles Pierre Léon.

Elle était sûrement très bonne chanteuse mais, comme Fouché, passionnée surtout par la musique du texte écrit. Elle était remarquable diseuse de textes en prose ou en vers, dont elle nous faisait découvrir toutes les subtilités. Mais, là aussi, on oubliait l'accentuation et l'intonation du parler ordinaire dont on n'a jamais extrait la moindre règle.

Comme tous les bons comédiens de cette époque, elle ignorait les aspects linguistiques qu'on aurait pu utiliser pour la correction phonétique. Ainsi, ayant constaté qu'un anglophone prononçait le mot français SITE, avec le I ouvert de l'anglais SIT, il aurait été plus rapide et plus efficace de lui montrer que tous les [i] du français se prononcent comme celui de SEAT en anglais, plutôt que de se servir de la célèbre phrase professeur: Mimi a mis ses amis à Miami, dont la séquence $m+i$ était censée assurer la bonne prononciation d'un [i] fermé.

Quoi qu'il en soit, Madame Mercier nous a appris beaucoup de trucs de correction phonétique, nous a fait chercher, trouver, retrouver des techniques efficaces. Elle nous a aussi appris à enseigner avec courtoisie, gentillesse, sourire. Nous répétant, dans son 
cours de pédagogie ce merveilleux conseil : «Ne dites jamais : Avez-vous compris?» mais : « Est-ce que c'est clair?»

Nous avons souvent regretté, Monique et moi et bien d'autres sans doute, de ne l'avoir pas mieux connue après l'avoir tant appréciée. Mais nous vivions à l'époque où le professeur avait une aura qui le rendait mystérieux. On le respectait, on le vénérait. On ne lui posait pas de questions sur sa vie, craignant souvent déjà d'en poser sur le travail.

Pourtant, elle nous a honorés de son amitié, nous donnant des conseils, nous prenant sous son aile. Quand nous avons enseigné à l'Institut de phonétique, nous la ramenions souvent chez elle, dans la modeste $4 \mathrm{CV}$ qu'elle nous avait aidés à acheter. Elle habitait boulevard Montparnasse, près de la Closerie des Lilas, où elle retrouverait sûrement quelques grands poètes. « Le monde est petit, mon petit ! Et l'entendre en parler était une merveille. On s'arrêtait devant chez elle. Après nous avoir remerciés avec effusion, elle ouvrait la portière, mettait le pied droit sur le trottoir, me prenait la main affectueusement le temps de redire combien elle nous aimait. Elle ne la lâchait plus. Une heure plus tard, nous étions toujours là, moi avec la main ankylosée! Mais c'était toujours de grands moments.

Nous devons à Fouché beaucoup de choses, en particulier le fait qu'il nous avait remarqués Monique et moi, à cause de notre passion pour la phonétique. Nous étions parmi les rares à oser lui poser des questions. Un jour, ayant besoin de personnel à l'Institut de phonétique, puis à l'École, il nous avait pris comme enseignants. Il lui paraissait que nous nous ressemblions et il nous appelait les jumeaux, jusqu'au jour où Arveiller lui dit : «Vos jumeaux, ils ont fait un beau coup. Ils viennent de se marier ! » Malgré ma reconnaissance pour le Maître, je ne résiste pas à citer la rencontre que j'ai eue avec lui, à la fin de ma licence ${ }^{1}$, et qui en dit long sur la mentalité des grands pontes de cette époque. J'avais réussi cette licence tout en étudiant à l'École. C'était bien, me dit-il, mais :

30 - Et maintenant?

31 - Maintenant, j'aimerais faire un doctorat.

$32-$ Vous le pouvez. Mais en quoi?

33 - En phonétique.

34 - Eh! Non, Léon, la phonétique, je m’en occupe.

\section{NOTES}

1. J'avais fait cette licence sur les conseils de Bernard Quemada, qui m'avait montré que, à cette époque-là, le diplôme de l'ESPPPFE n'était pas reconnu par l'Éducation nationale. C'est lui aussi qui m'a incité, quand j'enseignais aux États-Unis, à écrire ma première thèse sur la phonétique et la linguistique appliquée alors inconnue en France. Il m'a, par la suite, invité comme maîtreassistant à participer à la merveilleuse aventure du Centre de linguistique appliquée de la faculté des lettres de l'université de Besançon. Je lui garde, pour ça aussi, une bien grande reconnaissance. 
Faut-il ajouter que, après avoir feuilleté ma thèse de doctorat d'université, Fouché m'a dit: «Vous avez bien travaillé. Je vous félicite. Mais maintenant, Léon, faites des choses sérieuses »?

\section{RÉSUMÉS}

Monique et Pierre Léon, qui ont été formés à l'ESPPPFE juste après la Seconde Guerre mondiale, évoquent deux de ses figures, parmi les plus marquantes: celles de Pierre Fouché et de son assistante, Suzanne Mercier. Nommé directeur de l'École en 1944, Fouché y dispensait des cours d'orthoépie (principalement destinés aux étudiants étrangers), de philologie romane et de "phonétique générale du français ». Ancien élève de Maurice Grammont, refusant de discuter les questions théoriques de la phonologie (telles que les envisageait alors, en France, un Martinet) ou de s'intéresser aux innovations techniques de la phonétique expérimentale, Fouché consacrait l'un de ses cours aux faits de stylistique orale, à ce que l'on ne nommait pas encore phonostylistique, appliquée principalement aux textes littéraires. Formée au Conservatoire à la diction du théâtre, Suzanne Mercier donnait à l'École des leçons de correction phonétique en vue desquelles elle avait mis au point des techniques articulatoires originales. Grande diseuse de textes, elle négligeait les éclairages de la linguistique en matière de correction phonétique. Remarquables pédagogues l'un et l'autre, Fouché et Mercier se signalaient d'abord par leur art consommé de la maïeutique.

Monique and Pierre Léon, who were trained at the ESPPPFE just after WWII, draw attention to two most remarkable personalities: Pierre Fouché and his assistant Suzanne Mercier. Fouché became director of the School in 1944 and was teaching orthoepy (mainly to foreign students), Roman philology and "general French phonetics". Maurice Grammont's former student refused to discuss theoretical questions on phonology (unlike Martinet did at the time) or to get involved in any technical innovation in experimental phonetics. He would devote his classes to oral stylistics, that would later be called phonostylistics,mainly applied to literary texts. Suzanne Mercier, for her part, was trained at the 'Conservatoire d'art dramatique' (a drama school). She was in charge of phonetic correction for which she had developed interesting articulatory techniques. Being a brilliant text reader, she neglected the linguistic light on phonetic correction. Both were remarkable teachers and distinguished themselves mainly by their mastering of maieutics.

\section{INDEX}

Mots-clés : articulatory techniques, correction phonétique, EPPFE, ESPPPFE, Fouché (Pierre), Mercier (Suzanne), oral stylistics, phonetic correction, phonostylistics, phonostylistique, stylistique orale, techniques articulatoires

\section{AUTEURS}

\section{PIERRE LÉON}

Université de Toronto 


\section{MONIQUE LÉON}

Université de Toronto 